Int. J. Dev. Biol. 49: 539-546 (2005)

doi: $10.1387 / \mathrm{ijdb} .041922 \mathrm{dr}$

\title{
Regulation of phyllotaxis
}

\author{
DIDIER REINHARDT* \\ Plant Biology, Department of Biology, Fribourg, Switzerland
}

\begin{abstract}
Plant architecture is characterized by a high degree of regularity. Leaves, flowers and floral organs are arranged in regular patterns, a phenomenon referred to as phyllotaxis. Regular phyllotaxis is found in virtually all higher plants, from mosses, over ferns, to gymnosperms and angiosperms. Due to its remarkable precision, its beauty and its accessibility, phyllotaxis has for centuries been the object of admiration and scientific examination. There have been numerous hypotheses to explain the nature of the mechanistic principle behind phyllotaxis, however, not all of them have been amenable to experimental examination. This is due mainly to the delicacy and small size of the shoot apical meristem, where plant organs are formed and the phyllotactic patterns are laid down. Recently, the combination of genetics, molecular tools and micromanipulation has resulted in the identification of auxin as a central player in organ formation and positioning. This paper discusses some aspects of phyllotactic patterns found in nature and summarizes our current understanding of the regulatory mechanism behind phyllotaxis.
\end{abstract}

KEY WORDS: phyllotaxis, meristem, auxin, Arabidopsis, tomato

\section{Introduction}

The architecture of plants is highly regular. For example, flowers exhibit characteristic symmetric arrangement of their organs; the sepals, petals, anthers and carpels. Since this arrangement is precisely regulated, it has been used, besides other traits such as the shape and colour of floral organs, for taxonomic analysis. However, regular organisation is not only found in flowers, but also throughout vegetative development. The leaves are arranged in characteristic patterns, a phenomenon referred to as phyllotaxis (Schwabe, 1984; Steeves and Sussex, 1989; Reinhardt and Kuhlemeier, 2002). Phyllotaxis is a biological phenomenon that is accessible to anybody without expensive equipment. It is therefore not surprising that the beauty and regularity of phyllotactic patterns has been examined since classical antiquity (Adler et al., 1997).

Phyllotaxis is determined by the spatial and temporal regulation of leaf formation at the shoot apical meristem (Steeves and Sussex, 1989; Reinhardt and Kuhlemeier, 2002). The most prevalent phyllotactic patterns are distichous (alternate) or spiral if one primordium is formed at a time and decussate (opposite) or bijugate if primordia are formed in pairs (Fig. 1). In the case of decussate phyllotaxis, the leaf pairs are diverged by $90^{\circ}$, resulting in the formation of 4 vertical rows of leaves, whereas in bijugate phyllotaxis, the leaf pairs are diverged by approximately $68^{\circ}$, resulting in the generation of double spirals. Higher order whorled and multijugate systems result if three or more primordia are formed simultaneously. Spiral phyllotaxis is the most common pattern and is found in model plant species such as Arabidopsis, tobacco and tomato. An important model system for the study of distichous phyllotaxis is maize. Phyllotactic patterns can change during plant ontogeny. For example, most dicotyledonous plants exhibit a transition from the initial decussate phyllotaxis laid down during embryogenesis to spiral phyllotaxis during vegetative or floral development and ultimately to whorled phyllotaxis in the flower. Genetic evidence suggests that an auxin-related mechanism is involved in the formation and positioning of the cotyledons and the leaves, as well as of floral organs (Okada et al., 1991; Hardtke and Berleth, 1998; Reinhardt et al., 2000; Christensen et al., 2000; Benjamins et al., 2001;Pfluger and Zambryski, 2004). This indicates that the same organogenic mechanism may operate at all stages of development and when different phyllotactic patterns are observed during ontogeny. A common basis for different phyllotactic patterns is supported by cases in which different branches of an individual plant show fundamentally different phyllotactic patterns. For instance, decussate plants occasionally form branches with spiral or tricussate phyllotaxis when one or three primordia are formed per node, instead of two (Fig. 2). A remarkable case is represented by Magnolia species which exhibit a diversity of phyllotactic solutions in a population of trees and even between the branches of an individual tree (Zagorska-Marek, 1994). Furthermore, the monocot maize, like tomato and Arabidopsis, exhibits an auxin-dependent mechanism for organ

\footnotetext{
*Address correspondence to: Dr. Didier Reinhardt. Plant Biology, Department of Biology, Rue Albert Gockel 3, CH-1700, Fribourg, Switzerland. Fax: +41-26-300-9740. e-mail: didier.reinhardt@unifr.ch
} 
formation (Scanlon, 2003) indicating that a conserved auxinrelated mechanism may regulate phyllotaxis in all flowering plants.

Phyllotaxis has been a field of intense interest for centuries. Numerous theories, based on geometrical, biophysical and biochemical principles, have been formulated to explain phyllotaxis (reviewed in Schwabe, 1984; Steeves and Sussex, 1989; Adler et al., 1997; Reinhardt and Kuhlemeier, 2002). The present review gives an overview over the most recent development in the phyllotaxis field, with the emphasis on the role of auxin in organ formation and positioning.

\section{The origin of phyllotaxis}

In monocot plants, bilateral symmetry is established with the initiation of the scutellum, the single cotyledon. In the grasses, distichous (alternate) phyllotaxis is established and the bilateral symmetry is maintained during the entire life-span including the reproductive phase. Other monocots undergo a transition to spiral phyllotaxis either during vegetative development like the Liliaceae, Bromeliaceae, Agavaceae and Arecacea (=palm trees), or at the onset of flowering (Orchidaceae). Conversely, in many Bromeliaceae, the spiral pattern returns to distichous at the onset of flowering.

In dicot plants, bilateral symmetry is established with the initiation of the cotyledons which are formed as an opposite pair. The two first true leaves are also formed as an opposite pair that is diverged from the cotyledons by $90^{\circ}$. Hence, dicots start with

A
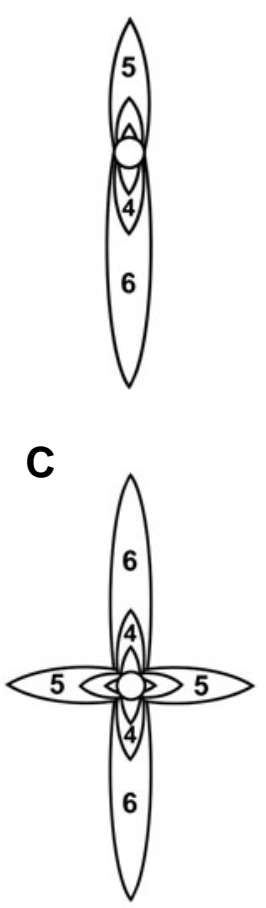

D

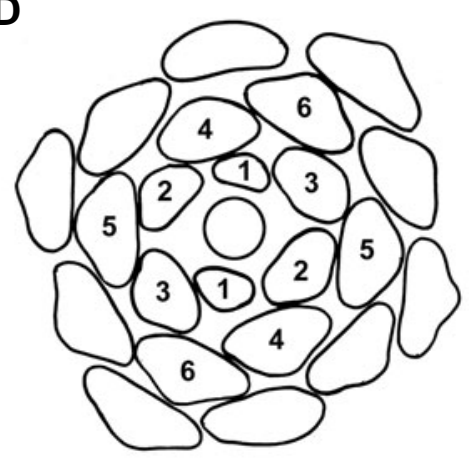

Fig. 1. Phyllotactic patterns in plants. (A) Distichous, (B) spiral (redrawn from Williams, 1974).; (C) decussate, (D) bijugate (redrawn from a picture provided by Alicja Banasiak). Primordia are numbered according to their age with the youngest as number 1. Note that in the text primordia are numbered as $P 1, P 2$ etc. The site of incipient leafformation $\left(L_{1}\right)$ is indicated by a star in (B). Circles in the centre represent the shoot apical meristem. decussate phyllotaxis. In some herbs (Lamiaceae), shrubs and trees (Aceraceae, Oleaceae) the decussate pattern is maintained, but the majority of plants undergoes a transition to spiral phyllotaxis during vegetative development or at the onset of flowering. The transition is gradual and can start as early as with the second or third pair of true leaves, if they are not formed in exactly opposite positions (Williams, 1974; Medford etal., 1992). This asymmetrical situation forces the next (single) leaf to be formed in the larger gap of the previous leaf pair and the resulting asymmetry is propagated to produce the stable spiral phyllotaxis found in the majority of higher plants. Since it has been the best-studied pattern, this review focuses on spiral phyllotaxis.

\section{Spiral phyllotaxis}

In spiral phyllotaxis, organs are formed with a constant divergence angle, usually $137.5^{\circ}$, resulting in an ontogenetic spiral which runs in clockwise or counter-clockwise direction (Fig. 1B). In addition, secondary spirals, the contact parastichies, can be discerned which run in both directions (Fig. 3). Curiously, the number of parastichies in each direction represents consecutive terms in the Fibonacci series (Steeves and Sussex, 1989). This series consists of terms that represent the sum of the previous two numbers $(0,1,1,2,3,5,8,13,21,34,55$, etc.). The number of contact parastichies depends on the relative size of the leaf primordia and the meristem. If the leaves are relatively large like in tomato, the phyllotactic pattern is characterized by low numbers such as 2:3. In a sunflower capitulum, in which the florets are small relative to the large flower disc, the numbers can be as high as 34:55. In many plants, the diameter of the meristem increases during development. This results in a gradual shift from lower to higher phyllotactic numbers, for example in flax, the phyllotactic pattern of the seedling starts with 3:5 and undergoes a gradual shift to $5: 8$ phyllotaxis (Fig. 3; Williams, 1975). Interestingly, the $3: 5$ pattern remains recognizable for some time, even after the 5:8 spirals are already clearly prominent (Fig. 3).

The term phyllotaxis is frequently used to refer to the final arrangement of organs (which I prefer to call the phyllotactic pattern). However, the term phyllotaxis is also used to describe the process of organ initiation and positioning at the meristem flank. Although the two phenomena are obviously related, they should be clearly distinguished. The phyllotactic patterns originally generated by the meristem can become altered in an expanding shoot system, thus the final phyllotactic pattern may not always reflect the original pattern of organ initiation. Here, I would like to focus more on the mechanisms involved in leaf positioning at the meristem flank, than on the astonishing precision of the final phyllotactic patterns.

\section{Phyllotaxis involves interactions between primordia and the meristem}

It has long been postulated that the positioning of a new leaf involves negative influences from older primordia (reviewed in: Steeves and Sussex, 1989). This influence could for instance be exerted by a diffusible inhibitor of organogenesis which suppresses leaf formation in the vicinity of existing primordia. According to this idea, the concentration of the inhibitor would decrease with increasing distance from the primordium, hence forming a 
Fig. 2. Branches of one individual Ailanthus altissima tree with different phyllotactic patterns. (A) Decussate, (B) tricussate, (C) spiral. Leaves in (C) are numbered with 1 being the youngest (note that this numbering does not correspond to the numbering of primordia in Fig. 1, since several unvisible primordia are not included here). (Modified from Reinhardtand Kuhlemeier, 2002)
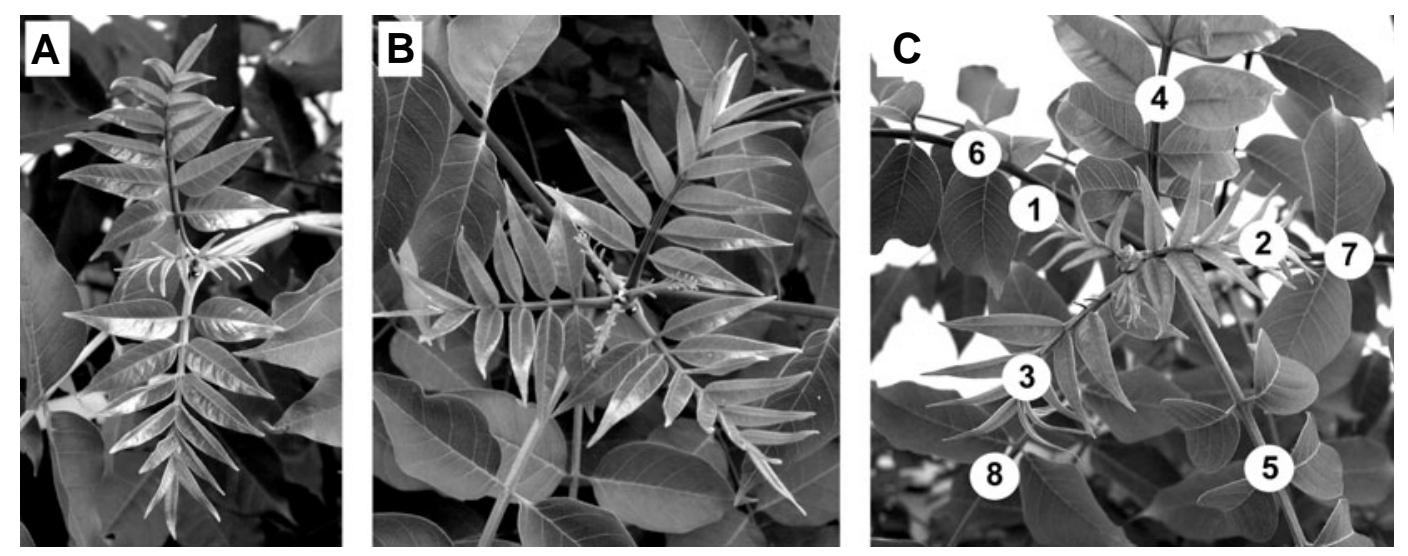

gradient around each primordium. At a characteristic distance, the concentration of the inhibitor would fall below a critical threshold, thus allowing leaf formation to occur. In its simplest form, this attractive model is based merely on a negative regulator of phyllotaxis and requires no specific inducer of leaf formation.

A new leaf $\left(L_{1}\right)$ is formed with the characteristic divergence angle of $137^{\circ}$ from the next older leaf in the ontogenetic spiral $\left(P_{1}\right)$. At the same time, the new leaf has characteristic distances towards its direct contact neighbours in the contact parastichies. In the case of a 3:5 system this is $P_{3}$ and $P_{5}$ (Fig. 1B). The questions therefore arises to what degree these three primordia contribute to the positioning of $\mathrm{L}_{1}$. Is the position of $\mathrm{L}_{1}$ determined at the level of the ontogenetic spiral (that is relative to $\mathrm{P}_{1}, \mathrm{P}_{2}$ etc) or according to its direct neighbours (that is relative to $P_{3}$ and $P_{5}$ in our example)? In a large phyllotactic system as the sunflower capitulum, it is more likely that the direct neighbours (e.g. $\mathrm{P}_{21}$ and $\mathrm{P}_{34}$ in a 21:34 system) can influence the positioning of $L_{1}$ than $P_{1}$ which is located at a considerable distance corresponding to nearly the entire diameter of the capitulum. On the other hand, in the small meristem of an Arabidopsis seedling, $\mathrm{L}_{1}$ is formed less than $100 \mu \mathrm{m}$ from $\mathrm{P}_{1}$. Thus, a negative influence from $P_{1}$ could easily be envisaged. Early classical work indicated that the position and the width of primordia is determined by the contact neighbours (Snow and Snow, 1931). In these experiments, primordia were separated from the meristem by tangential incisions. This resulted in the displacement of the future contact neighbours towards the site of the isolated primordium. Recently, these findings have been complemented by experiments which showed that in tomato, meristems that were isolated from the rest of the shoot (together with the smallest visible primordium) continued their correct ontogenetic spiral (Reinhardt et al., in press). Hence, the one preexisting primordium (possibly together with the next already determined primordium), provided enough information to maintain the ontogenetic spiral, thus, the direct contact neighbours are not absolutely required for spiral phyllotaxis in tomato. However, the new primordia grew much larger than normal, indicating that the preexisting direct neighbours normally determine the lateral extension of new primordia and thus their exact final size and position (Reinhardt et al., 2005).

\section{Auxin - the trigger of organ formation}

Two mutants have been described in Arabidopsis with serious defects in organogenesis. Their shoot axis grows as a naked stem, hence their names pin formed1 (pin1 ; Okada et al., 1991) and pinoid (pid; Bennett et al., 1995). A third mutant, monopteros (mp ; Hardtke and Berleth, 1996), which got its name from the fused cotyledons (Mayer et al., 1991), likewise exhibits a defect in leaf and flower formation (Przemeck et al., 1996). Interestingly, pin1, pid and mp mutants show organogenetic phenotypes at all stages of development. During embryogenesis, the cotyledons are fused and oversized ( $p$ in 1 and $m p$ ), or supernumerary cotyledons are formed (pid). Vegetative phenotypes include oversized and fused leaves (pin1), aberrant leaf positioning ( $p$ in 1, $m p$ ) and increased leaf number (pid). All three mutants exhibit strong flower phenotypes (organ number, position and identity), resulting in complete sterility. These results show that the same mechanism, mediated by $P I N 1, P I D$ and $M P$, is involved in organ formation and positioning at all stages of development. PIN1, PID and MP function in the transport of and response to the plant hormone auxin (Gälweiler $e t$ al., 1998; Christensen, 2000; Benjamins, 2001; Hardtke and Berleth, 1998). Recent studies showed that chemical inhibitors of the auxin tranporters also abolished organ formation and that application of exogenous auxin could restore organ formation both on the mutants or chemically treated pin structures (Reinhardt et al., 2000). In addition, the auxin transport regulator PIN1 is required for the correct spatial expression of organ and boundary marker genes (Vernoux et al., 2000). These results established auxin as the trigger of leaf and flower formation and as a component of phyllotactic patterning.

\section{A role for auxin in phyllotaxis}

The discovery that auxin acts as a trigger of organ formation opened the possibility that phyllotaxis is regulated by the distribution of auxin in the meristem. It is possible that auxin accumulates at the position of incipient organ formation, but not at positions between organs (Kuhlemeier and Reinhardt, 2001). In this case, auxin would not simply act as a permissive factor required for organogenesis, but as an instructive signal molecule. This view is supported by the fact that exogenous auxin triggers ectopic organs in tomato meristems (Reinhardt et al., 2000). The (indirect) conclusion of this experiment is that auxin distribution has to be regulated not only to permit organ formation, but also to restrict it to the correct sites. Since the preformed primordia influence the positioning of new organs (see above), the accumulation of auxin in the meristem may be expected to be influenced by the primordia. One possibility would be that they mediate the transport of auxin specifically to the site of organ initiation. An alternative possibility would be that the 
primordia influence the distribution of auxin in the meristem by absorbing it from their surroundings (Reinhardt and Kuhlemeier, 2002). The latter scenario could explain the regular spacing, if the efficiency of absorption declines with the distance from the primordium, such that auxin accumulation in the meristem can occur only at characteristic minimal distances from preexisting primordia. Thus, the primordia would actually have the opposite function of the one proposed in the inhibitor model. Instead of releasing an inhibitor of leaf formation, they absorb an activator (auxin) from the meristem. However, both mechanisms would lead to similar characteristic spacing of leaves.

\section{Auxin transport proteins generate phyllotactic patterns}

Auxin is transported through the tissues by cellular import and export proteins (Lomax et al., 1995). Long-distance transport may also take place in the vasculature (Swarup et al., 2001), but in the undifferentiated tissues of the shoot apex, this can be excluded. Genetic analysis has shown that central components of auxin tranport are represented by the putative influx carrier AUXIN RESISTANT1 (AUX1; Bennett et al., 1996) and the putative efflux carrier PIN1 (Gälweiler et al., 1998). AUX1 is a member of a gene family together with LIKE AUX1 (LAX1), LAX2 and LAX3 (Parry et al., 2001), whereas PIN1 is part of a family of eight members (Friml and Palme, 2002). Although AUX1 and PIN1 may require additional factors for effective auxin transport, they are central components of the transport mechanism and their subcellular localisation is compatible with an involvement in auxin influx and efflux, respectively. Therefore, I will, for simplicity, refer to AUX1 and PIN1 as the influx and efflux carrier, respectively, although their precise biochemical function in polar auxin transport remains to be identified.

The requirement for auxin transport for organ formation (Okada et al., 1991; Reinhardt et al., 2000) highlighted the need to determine the expression and subcellular localisation of the auxin transporters, in order to predict the direction of auxin fluxes in the shoot apex. A recent study describes the expression and subcel- lular localisation of AUX1 and PIN1 in the shoot apex of Arabidopsis and provides evidence for the hypothesis that primordia act as auxin sinks (Reinhardt et al., 2003).

The influx carrier AUX1 is expressed in the external $L_{1}$ layer of the meristem (representing the skin of the meristem) and in the abaxial epidermis of the developing primordia. Therefore, auxin can be expected to accumulate in these cells. PIN1 is also expressed primarily in the cells of the $L_{1}$ layer, but it is restricted to the side of the cells that points to the meristem centre. Hence, the auxin which is accumulated in the $L_{1}$ layer by AUX1 appears to be transported up towards the centre of the meristem dome. This can explain how the meristem is supplied with auxin, but how does auxin accumulate at the right place to induce a leaf? Interestingly, PIN1 is induced in incipient and young primordia, where it is localized to the side of the cells that points to the centre of the primordia. This indicates the local transport of auxin to the centre of the primordia. Later, PIN1 expression in the primordia becomes restricted to a narrow cell file along the central axis (probably corresponding to the developing mid-rib) and becomes localised to the lower side of the cells, as in the stem (Gälweiler et al., 1998), indicative of downwards transport through the centre of the primordia. These findings are compatible with a sink function of primordia as proposed in the " auxin-sink « model of phyllotaxis (Reinhardt et al., 2003; Figure 4).

A role for active auxin transport in meristem patterning is inferred from the loss of phyllotactic expression patterns of diagnostic marker genes for meristem, organ and boundary identity (Vernoux et al., 2000; Scanlon, 2003). More directly, it was shown that the unpatterned meristem of pinoid mutants can be induced to form a whorl of separated, evenly spaced primordia if supplied with the natural auxin IAA (Reinhardt et al., 2003). The generation of this pattern was dependent on the PIN1 gene. Moreover, the supply with the synthetic auxin analogue 2,4-D, which is not a substrate for PIN1, did not induce a similar pattern. This shows that active polar auxin transport, mediated through PIN1, is necessary for meristem patterning and can generate de novo patterns.

Taken together, these data indicate that auxin is transported in the meristem primarily through the $\mathrm{L}_{1}$ layer and becomes redistributed within the meristem by the primordia such that accumulation is prevented in the vicinity of developing primordia. At a certain distance from the primordia, sufficient auxin can accumulate to allow a new primordium to be formed. This will itself start to function as a sink to regulate further phyllotaxis, but it will remain in competition with its direct neighbours, which may serve to restrict its lateral extension.

\section{What regulates PIN1 expression and localisation?}

The regulation of PIN1 expression appears to respond to different inputs. For example, expression in the $L_{1}$ layer of the meristem is likely a response to cell type specific cues. On the other hand, PIN1 is upregulated in young primordia and its
Fig. 3. Spiral phyllotaxis in flax. Left: 11 days after germination, the phyllotactic pattern is characterized by 3 counter-clockwise parastichies (one represented in red) and 5 clockwise parastichies (3:5 phyllotaxis). 11 days later, as a result of the increase in the size of the apex, the phyllotactic pattern has shifted to 5:8. However, the original 3 parastichies are still evident (one represented in red). (modified from Williams, 1974).

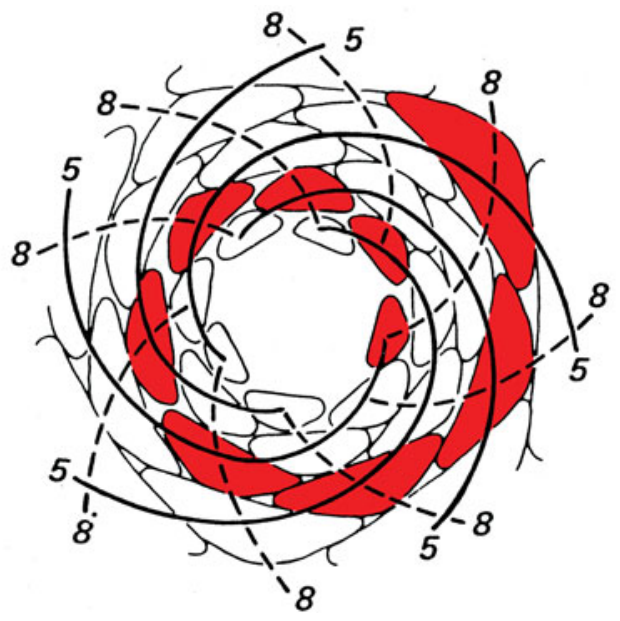


expression is induced by auxin (Reinhardt et al., 2003). This latter effect means that auxin is both upstream (as a regulator) and downstream (as a substrate) of PIN1. In the context of phyllotaxis, auxin may therefore mediate its reiterative nature: PIN1 activity in the preexisting primordia leads to auxin accumulation at $L_{1}$, which in turn induces PIN1 to ensure proper positioning of $L_{2}$ and so on.

How is the subcellular localisation of PIN1 regulated? In the globular embryo, PIN1 is first expressed in all cells and does not exhibit a particular subcellular localisation (Steinmann etal., 1999). The first sign of PIN1 polarisation can be observed in the central cells just when they become axialised and elongate in the apicalbasal dimension. In the gnom mutant, this process and the later regulation of PIN1 localisation is disturbed. GNOM encodes a regulator of GTPases that are involved in vesicle trafficking (Geldner, 2004). These results correlate with the finding that the inhibitor of vesicle trafficking, brefeldin A (BFA), abolishes PIN1 localisation and that well characterised auxin tranport inhibitors act by influencing vesicle trafficking (and consequently PIN1 localisation) rather then auxin transport itself (Geldner et al., 2001; 2003). These findings have resulted in the view that PIN1 is subject to continuous subcellular recycling between the plasmalemma and internal (endosomal) compartements. Hence the seemingly stable polar localization of PIN1 observed in immunocytochemical experiments masks rapid dynamic recycling of PIN1. Besides GNOM, which is a general component of vesicular trafficking, additional components with specificity for PIN1 can be expected to be involved. The question remains of how the vesicular trafficking system is informed where on the plasmalemma to unload its cargo. Considering the strong, stable nature of cell polarity in plants, the mechanism may well involve a self-reinforcing component. On the other hand, the dynamics of patterning in embryogenesis and phyllotaxis require rapid and reliable reorientation of auxin transporters. In the case of phyllotaxis, the PIN1-localising mechanism may respond to auxin fluxes. Such a positive feedback mechanism has been postulated to operate in auxin-mediated vascular patterning (Sachs, 1981; Berleth et al., 2000). It has been established that the routing of vascular strands, including the auxin conducting xylem parenchyma cells, respond to exogenous auxin. Thus, auxin can influence it's own route of transport.

\section{What's more?}

Leaves are formed in the peripheral zone (PZ) of the meristem which encircles the central zone (CZ), the site of the stem cells (Steeves and Sussex, 1989). It appears that this subdivision of the meristem is independent from the auxin-related phyllotactic patterning mechanism (Reinhardt et al., 2000). However, the circumference and the width of the PZ inevitably influence organ formation and phyllotaxis. It is therefore conceivable that phyllotaxis could be regulated not only directly by a mechanism acting on the rate, the range, or the direction of auxin flux, but indirectly, by controlling the size of the $\mathrm{PZ}$ or the size of the primordia. For example, an increase in the circumference of the apex could lead to the accomodation of more organs at a time. Interestingly, in the abphy/1 mutant of maize, the transition of distichous to decussate phyllotaxis is associated with an increase in meristem size (Jackson and Hake, 1999). The abphy/1 mutant carries a mutation in a putative cytokinin-sensor, indicating that phyllotaxis could be regulated by cytokinin-dependent control of meristem size (Giulini etal.,
2004). In the clavata and fasciata mutants, in which the circumference of the meristem is dramatically increased, phyllotaxis is also affected. In this case, however, phyllotaxis becomes irregular (Leyser and Furner, 1992; Clark et al., 1993, 1995).

It has been pointed out that the vertical spacing of primordia may be important to determine the range of influence from preexisting primordia onto the meristem (Schwabe, 1984). Indeed, chemical, as well as surgical treatments that lead to the elongation of the shoot apex are associated with phyllotactic changes from spiral to distichous (Schwabe, 1971; Reinhardt et al., 2005). This effect is compatible with the interpretation that, due to the vertical elongation, $\mathrm{P}_{2}$ became too remote from the meristem to exert an inhibitory influence and therefore, $L_{1}$ was formed opposite to $P_{1}$.

These examples show that phyllotaxis could be regulated not only directly by auxin transport, but by more general changes of meristem organization or tissue features which affect the range and/or the rate of auxin transport.

\section{Computer models of phyllotaxis}

Computer modelling has been employed to analyse phyllotactic patterns (Mitchison, 1977; Veen and Lindenmayer, 1977; Meinhardt, 1984; Green, 1992, 1996; Douady and Couder, 1996). Two major phyllotactic mechanisms have been envisaged. Biophysical models invoked forces such as tension, compression and shear as the principal parameters influencing phyllotaxis (Green, 1992, 1996). According to this idea, no differential regulation of cell identity and growth in the meristem is required. Instead, growth is regulated at a global level (the entire meristem) and patterns emerge as a consequence of local changes (e.g. at $L_{1}$ ) in biophysical param-
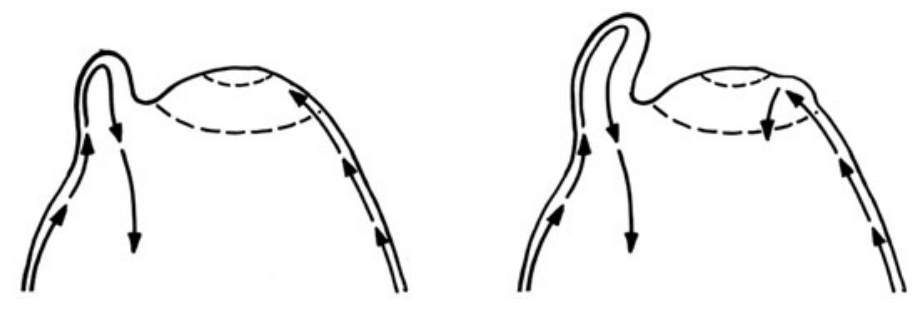

Fig. 4. A model for the regulation of phyllotaxis by auxin. (Left) In the meristem, auxin is acropetally transported by the $L_{1}$ layer. In the preexisting primordia (on the left), auxin is absorbed and transported basipetally to the stem (left). On the rigth side, auxin can reach the peripheral zone and induce organ formation. (Right) After induction of organ formation on the right side, a new sink is established that diverts auxin away from the meristem. Reproduced with permission from Reinhardt et al., 2003, (c) Nature Publishing Group.

eters that result from the growth and development of the apex as a whole (Green, 1992, 1996).

A contrasting view is represented by the majority of other models in which diffusible signal molecules are envisaged to regulate phyllotaxis (Mitchison, 1977; Veen and Lindenmayer, 1977; Meinhardt, 1984). The central component of most such models is an inhibitor of leaf formation which emanates from preexisting primordia to establish an inhibitory field. The superposition of all inhibitory fields defines the site of lowest inhibitor concentration. As the apex grows and expands, the preexisting primordia move apart and away from the meristem centre until a 


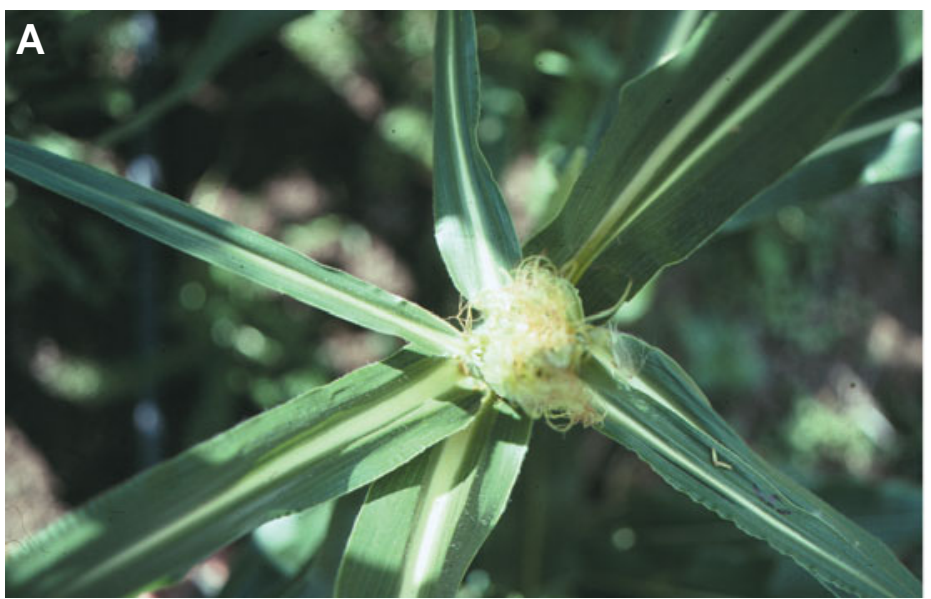

Fig. 5. Phyllotaxis in the terminal ear1 (te1) mutant of maize. (A) Top view of a te1 plant. Instead of distichous (alternate) phyllotaxis, te1 exhibits irregular phyllotaxis. (B) Lateral view of a te1 shoot axis with irregular internodes (leaves were removed). Reproduced with permission from Veit et al., 1998, (c) Nature Publishing Group.

threshold is reached at the site of lowest inhibitor concentration, allowing leaf formation to occur. Although such models can regenerate various phyllotactic patterns, there is no experimental support for the involvement of a diffusible inhibitor in phyllotaxis. The recent establishment of polar auxin transport as the central mechanism of phyllotaxis can now be used to develop new models for phyllotaxis which include all known parameters and therefore will give a more realistic representation of phyllotaxis. The development of such models will stimulate our critical thinking about the role of auxin and help to identify important issues that need to be approached experimentally.

\section{Why did regular phyllotaxis evolve?}

The dominance of regular phyllotactic patterns in nature suggests that there must be a selective advantage of regular versus random arrangement of organs. In the case of flowers, it is conceivable that regular architecture is important for the attraction of pollinators, but why would leaf position have to be regular? It has been proposed that spiral arrangements of leaves may optimize the absorption of the sunlight for photosynthesis (reviewed in Niklas, 1988). While this argument is not entirely convincing in the case of spiral phyllotaxis (Niklas, 1988), it certainly does not hold for distichous and decussate phyllotaxis. In these cases, the leaves are formed in two or four rows along the stem (Figure 1A,C) and therefore tend to shade each other. Also, leaf position is frequently adjusted post-meristematically, indicating that optimisation of light perception does not require precise patterns of leaf formation.

The occurrence of several distinct phyllotactic patterns in nature may indicate that regularity itself, rather than the specific phyllotactic arrange-ment, represents a selective advantage over random leaf arrangement. The study of leaf formation has taught us that the process of-leaf initiation is intimately linked with the phyllotactic positioning. Several mutants affected in the process of leaf formation also exhibit defects in leaf positioning (see above). Therefore, it may be the process of leaf formation at the meristem, rather then the function of the final arrangement of the mature foliage, that requires regularity for optimal function. Besides the formation of

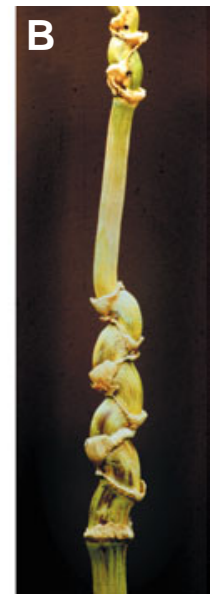

leaves and flowers, the meristem carries out at least two additional important functions, namely self-maintenance and the formation of the internodes. The stem cells required for self-maintenance reside in the central zone of the meristem, where they continuously produce new cell material for organogenesis. Founder cells are selected at specific sites in the peripheral zone. Thus, the meristem has to provide exactly the right number of cells at the right position and at the right time to replenish the cells engaged in organogenesis. Here, regular phyllotaxis may represent a selective advantage over random leaf position, allowing the'meristem to optimally allocate founder cells, thus avoiding depletion or over-proliferation of organogenic cells on one side of the apex. Since the meristem is responsible for the formation of the stem, an imbalance of the cell number could potentially affect shoot architecture as a whole. For example, if cells were depleted on one side, there would be less cells for the formation of the internode. The resulting internode would therefore be curved. In this context, it is interesting to note that mutants with irregular phyllotaxis frequently exhibit irregular and sometimes curved internodes (Okada etal., 1991; Veit et al., 1998; Figure 5). Thus, regular phyllotaxis may be important for optimal development at the shoot tip as well as for the final architecture of the entire shoot.

\section{Conclusions and Outlook}

Theoretical concepts of biological patterning frequently envisage mechanisms that are hierarchically organised. For example, in the development of the Drosophila embryo, the establishment of the anterior and posterior pole is followed by a sequential series of patterning events that finally produce the different specialized segments of the adult body in the correct spatial arrangement (Pick, 1998). In the case of $C$. elegans, the hierarchical organization of development is reflected in an invariant sequence of cell divisions and cell differentiation events, resulting in a stereotyped fate map for each individual cell of the body (Labouesse and Mango, 1999). Such a hierarchical view of development has led to the notion that separate mechanisms are responsible for patterning and later for the appropriate development of the elements of the pattern. In contrast to this view, phyllotaxis is not hierarchical but reiterative. This has important implications: the result of the patterning and the following developmental events feed continuously back onto the patterning mechanism. Therefore, phyllotaxis and the control of "downstream" events like leaf growth, differentiation and tissue polarization are intimately linked. Key questions for the future will be the nature of the feedback mechanisms involved. For example, how is the localization of the auxin transporters controlled? Is there a role for the substrate auxin in the localization of its own transporters? Further, it will be important to find out how auxin signals are interpreted by the cells (auxin concentration or flux?) and how these signals are translated into tissue growth, cell proliferation and differentiation.

\section{Acknowledgements}

I wish to thank Cris Kuhlemeier, Przemek Prusinkiewicz and Beata 
Zagorska-Marek for stimulating discussions and Sam Zeeman for critical reading of the manuscript.

\section{References}

ADLER, I., BARABE, D. and JEAN, R.V. (1997). A history of the study of phyllotaxis. Ann. Bot. 80: 231-244.

BENJAMINS, R., QUINT, A., WEIJERS, D., HOOYKAAS, P. and OFFRINGA, R. (2001). The PINOID protein kinase regulates organ development in Arabidopsis by enhancing polar auxin transport. Development 128, 4057-4067.

BENNETT, S.R.M., ALVAREZ, J., BOSSINGER, G. and SMYTH, D.R. (1995). Morphogenesis in pinoid mutants of Arabidopsis thaliana. Plant J. 8, 505-520.

BENNETT, M. J., MARCHANT, A., GREEN, H.G., MAY, S.T., WARD, S.P., MILLNER, P.A., WALKER, A.R., SCHULZ, B. and FELDMANN, K.A. (1996). Arabidopsis AUX1 gene: A permease-like regulator of root gravitropism. Science 273: 948950.

BERLETH, T., MATTSSON, J. and HARDTKE, C.S. (2000). Vascular continuity, cell axialisation and auxin. Plant Growth Regulation 32: 173-185.

CHRISTENSEN, S.K., DAGENAIS, N., CHORY, J. and WEIGEL, D. (2000). Regulation of auxin response by the protein kinase PINOID. Cel/ 100: 469-478.

CLARK, S.E., RUNNING, M.P. and MEYEROWITZ, E.M. (1993). CLAVATA1, a regulator of meristem and flower development in Arabidopsis. Development 119: 397-418.

CLARK, S.E., RUNNING, M.P. and MEYEROWITZ, E.M. (1995). CLAVATA3 is a specific regulator of shoot and floral meristem development affecting the same process as CLAVATA1. Development 121: 2057-2067.

DOUADY, S. and COUDER, Y. (1996). Phyllotaxis as a dynamical self organizing process. Part II: The spontaneous formation of a periodicity and the coexistence of spiral and whorled patterns. J. Theor. Biol. 178: 275-294.

FRIML, J. and PALME, K. (2002). Polar auxin transport - old questions and new concepts? Plant Mol Biol 49: 273-284.

GÄLWEILER, L., GUAN, C.H., MÜLLER, A., WISMAN, E., MENDGEN, K., YEPHREMOV, A. and PALME, K. (1998). Regulation of polar auxin transport by AtPIN1 in Arabidopsis vascular tissue. Science 282: 2226-2230.

GELDNER, N., FRIML, J., STIERHOF, Y.-D., JÜRGENS, G. and PALME, K. (2001) Auxin transport inhibitors block PIN1 cycling and vesicle trafficking. Nature 413: 425-428.

GELDNER, N. ANDERS, N., WOLTERS, H., KEICHER, J., KORNBERGER, W., MÜLLER, P., DELBARRE, A., UEDA, T., NAKANO, A. and JÜRGENS G. (2003). The Arabidopsis GNOM ARF-GEF mediates endosomal recycling, auxin transport and auxin-dependent plant growth. Cell 112: 219-230.

GELDNER, N. (2004). The plant endosomal system - its structure and role in signal transduction and plant development. Planta 219: 547-560.

GIULINI, A., WANG, J. and JACKSON, D. (2004). Control of phyllotaxy by the cytokinin-inducible response regulator homologue ABPHYL1. Nature 430:10311034.

GREEN, P.B. (1992). Pattern Formation in shoots: A likely role for minimal energy configurations of the tunica. Int. J. Plant Sci. 153: S59-S75.

GREEN, P.B. (1996). Expression of form and pattern in plants - a role for biophysical fields. Semin. Cell Dev. Biol. 7: 903-911.

HARDTKE, C.S. and BERLETH, T. (1998). The Arabidopsis gene MONOPTEROS encodes a transcription factor mediating embryo axis formation and vascular development. EMBO J. 17: 1405-1411.

JACKSON, D. and HAKE, S. (1999). Control of phyllotaxy in maize by the ABPHYL1 gene. Development 126: 315-323.

KUHLEMEIER, C. and REINHARDT, D. (2001). Auxin and phyllotaxis. Trends Plant Sci. 6: $187-189$

LABOUESSE, M. and MANGO, S.E. (1999). Patterning the C. elegans embryo moving beyond the cell lineage. Trends Genet. 15: 307-313.

LEYSER, H.M.O. and FURNER, I.J. (1992). Characterisation of three shoot apical meristem mutants of Arabidopsis thaliana. Development 116: 397-403.

LOMAX, T.L., MUDAY, G.K. and RUBERY, P.H. (1995). Auxin transport. in" Plant hormones: Physiology, biochemistry and molecular biology (ed. P.J. Davies) pp. 509-530. Kluwer Academic Press, Dordrecht, The Netherlands.
MAYER, U., TORRES-RUIZ, R.A., BERLETH, T., MISERA, S. and JÜRGENS, G. (1991). Mutations affecting body organization in the Arabidopsis embryo. Nature 353: 402-407.

MEDFORD, J.I., BEHRINGER, F.J., CALLOS, J.D. and FELDMANN, K.A. (1992). Normal and abnormal development in the Arabidopsis vegetative shoot apex. Plant Cell 4: 631-643.

MEINHARDT, H. (1984). Models of pattern formation and their application to plant development, in Positional Controls in Plant Development, (eds P.W. Barlow and D.J. Carr), pp. 1-32. Cambridge University Press, Cambridge, UK, 1-32.

MITCHISON, G.J. (1977). Phyllotaxis and the Fibonacci series. Science 196: 270275.

NIKLAS, K.J. (1988). The role of phyllotactic pattern as a "developmental constraint" on the interception of light by leaf surface. Evolution 42: 1-16.

OKADA, K., UEDA, J., KOMAKI, M.K., BELL, C.J. and SHIMURA, Y. (1991). Requirement of the auxin polar transport-system in early stages of Arabidopsis floral bud formation. Plant Cel/ 3: 677-684

PARRY, G., MARCHANT, A., MAY, S., SWARUP, R., SWARUP, K., JAMES, N., GRAHAM, N., ALLEN, T., MARTUCCI, T., YEMM, A., NAPIER, R., MANNING, K., KING, G. and BENNETT, M. (2001). Quick on the uptake: Characterization of a family of plant auxin influx carriers. J. Plant Growth Regul. 20, 217-225.

PFLUGER, J. and ZAMBRYSKI, P. (2004). The role of SEUSS in auxin response and floral organ patterning. Development 131: 4697-4707.

PICK, L. (1998). Segmentation: Painting stripes from flies to vertebrates. Dev. Genetics 23: 1-10.

PRZEMECK G.K.H., MATTSSON J., HARDTKE C.S., SUNG Z.R. and BERLETH T. (1996). Studies on the role of the Arabidopsis gene MONOPTEROS in vascular development and plant cell axialization. Planta 200:229-237.

REINHARDT, D., MANDEL, T. and KUHLEMEIER, C. (2000). Auxin regulates the initiation and radial position of plant lateral organs. Plant Cel/ 12: 507-518.

REINHARDT, D. and KUHLEMEIER, C. (2002). Phyllotaxis in higher plants. in Meristematic tissues in plant growth and development (eds. M.T. McManus and B.E. Veit) Sheffield Academic Press Ltd, Sheffield, UK.

REINHARDT, D., PESCE, E.-R., STIEGER, P., MANDEL, T., BALTENSPERGER, K., BENNETT, M., TRAAS, J., FRIML, J. and KUHLEMEIER, C. (2003). Regulation of phyllotaxis by polar auxin transport. Nature 426: 255-260.

REINHARDT, D., FRENZ, M., MANDEL, T. and KUHLEMEIER C. Microsurgical and laser ablation analysis of leaf positioning and dorsoventral patterning in tomato, Development, 132: 15-26.

SACHS, T. (1981). The control of patterned differentiation of vascular tissues. Advances in Botanical Research 9: 151-262.

SCANLON, M.J. (2003). The polar auxin transport inhibitor N-1-naphthylphthalamic acid disrupts leaf initiation, KNOX protein regulation and formation of leaf margins in maize. Plant Physiol. 133: 597-605.

SCHWABE, W.W. (1971). Chemical modification of phyllotaxis and its implications. Symp. Soc Exp Biol 25: 301-322.

SCHWABE, W.W. (1984). Phyllotaxis. in Positional Controls in Plant Development (eds. P.W. Barlow and D.J. Carr) pp. 403-440. Cambridge University Press, Cambridge, UK.

SNOW, M. and SNOW, R. (1931). Experiments on phyllotaxis.I. The effect of isolating a primordium. Phil Trans $R$ Soc Lond $B$ 221: 1-43.

STEEVES, T.A. \& SUSSEX, I.M. (1989). Patterns in plant development (Cambridge University Press, Cambridge, UK.

STEINMANN T., GELDNER N., GREBE M., MANGOLD S., JACKSON C.L., PARIS S., GÄLWEILER L., PALME K., JÜRGENS G. (1999). Coordinated polar localization of auxin efflux carrier PIN1 by GNOM ARF GEF. Science 286: 316-318.

SWARUP, R., FRIML, J., MARCHANT, A., LJUNG, K., SANDBERG, G., PALME, K. and BENNETT, M. (2001). Localization of the auxin permease AUX1 suggests two functionally distinct hormone transport pathways operate in the Arabidopsis root apex. Genes \& Development 15: 2648-2653.

VEEN, A. H. and LINDENMAYER, A. (1977). Diffusion mechanism for phyllotaxis. Theoretical, physico-chemical and computer study. Plant Physiol. 60: 127-139.

VEIT, B., BRIGGS, S.P., SCHMIDT, R.J., YANOFSKY, M.F. AND HAKE, S. (1998). Regulation of leaf initiation by Terminal Ear1 gene of maize. Nature 393: 166-168. VERNOUX, T., KRONENBERGER, J., GRANDJEAN, O., LAUFS, P. and TRAAS, J. 
(2000). PIN-FORMED 1 regulates cell fate at the periphery of the shoot apical meristem. Development 127: 5157-5165.

WILLIAMS, R.F. (1975). The shoot apex and leaf growth. Cambridge University
Press, Cambridge.

ZAGORSKA-MAREK, B. (1994). Phyllotaxic diversity in Magnolia flowers. Act. Soc. Bot. Pol. 63: 117-137. 